\title{
The significance of different diacylgycerol synthesis pathways on plant oil composition and bioengineering
}

\author{
Philip D. Bates and John Browse* \\ Institute of Biological Chemistry, Washington State University, Pullman, WA, USA
}

Edited by:

Kazuki Saito, Chiba University, Japan

Reviewed by:

Kent D. Chapman, University of North

Texas, USA

Ikuo Nishida, Saitama University,

Japan

*Correspondence:

John Browse, Institute of Biological

Chemistry, Washington State

University, Clark Hall, Pullman, WA

99164-6340, USA.

e-mail: jab@wsu.edu
The unique properties of vegetable oils from different plants utilized for food, industrial feedstocks, and fuel is dependent on the fatty acid (FA) composition of triacylglycerol (TAG). Plants can use two main pathways to produce diacylglycerol (DAG), the immediate precursor molecule to TAG synthesis: (1) De novo DAG synthesis, and (2) conversion of the membrane lipid phosphatidylcholine (PC) to DAG. The FA esterified to PC are also the substrate for FA modification (e.g., desaturation, hydroxylation, etc.), such that the FA composition of PC-derived DAG can be substantially different than that of de novo DAG. Since DAG provides two of the three FA in TAG, the relative flux of TAG synthesis from de novo DAG or PC-derived DAG can greatly affect the final oil FA composition. Here we review how the fluxes through these two alternate pathways of DAG/TAG synthesis are determined and present evidence that suggests which pathway is utilized in different plants. Additionally, we present examples of how the endogenous DAG synthesis pathway in a transgenic host plant can produce bottlenecks for engineering of plant oil FA composition, and discuss alternative strategies to overcome these bottlenecks to produce crop plants with designer vegetable oil compositions.

Keywords: diacylglycerol, triacylglycerol, phosphatidylcholine, fatty acid, acyl editing, biotechnology, oilseed, hydroxylase

\section{INTRODUCTION}

Triacylglycerol (TAG) that accumulates in seeds or fruits of plants is a major renewable source of reduced carbon utilized as food, industrial feedstocks, and fuel. The function and value of different plant oils is derived from the fatty acid (FA) composition of TAG. The major vegetable oil crops (soybean, palm, canola, sunflower) have FA compositions limited to primarily five FA. Saturates 16:0 and 18:0, monounsaturated 18:1 $\Delta 9$, and polyunsatured FA (PUFA) 18:2 $\Delta 9,12$ and 18:3 $\Delta 9,12,15$ (\# carbons:\# double bonds, $\Delta$ double bond position; Table 1). Nevertheless, within the plant kingdom there are $>300 \mathrm{FA}$ structures that differ in chain length, number and position of double bonds, or that contain different functional groups such as hydroxy, epoxy, etc. (Badami and Patil, 1980). These unusual FAs have many uses as industrial feedstocks for lubricants, polymers, and resins. However, most plants that produce unusual FAs are either limited in oil yield or have undesirable agronomic features (Badami and Patil, 1980; Voelker and Kinney, 2001; Dyer et al., 2008). With very few exceptions (Knutzon et al., 1999; Nguyen et al., 2010), attempts to engineer oilseed plants to accumulate unusual FAs has resulted in low yields of the unusual FA in seed TAG, especially the engineering of unusual FAs synthesized within membrane lipids (Cahoon et al., 2007; Dyer et al., 2008; Lu et al., 2011). The limited successes in oilseed engineering highlight the fact that we still do not fully understand how plants accumulate oils with very different FA compositions, even though the enzymes, genetics, and regulation of FA and TAG synthesis have been extensively studied (Dyer et al., 2008; Snyder et al., 2009; Weselake et al.,
2009; Baud and Lepiniec, 2010; Li-Beisson et al., 2010; Napier and Graham, 2010; Wallis and Browse, 2010; Lu et al., 2011; Chapman and Ohlrogge, 2012). Of particular importance is that plants can utilize at least two metabolic pathways to produce different molecular species of the immediate precursor to TAG, diacylglycerol (DAG): (1) De novo DAG synthesis, and (2) conversion of the membrane lipid phosphatidylcholine (PC) to DAG. The FA esterified to PC are also the substrate for FA modification (e.g., desaturation, hydroxylation, etc.), such that the FA composition of PC-derived DAG can be substantially different than that of de novo DAG, and ultimately affect two-thirds of the TAG FA composition.

In this review we outline various possible metabolic pathways to produce DAG and subsequent TAG with different FA compositions: de novo DAG synthesis, de novo DAG synthesis combined with PC-FA modification and acyl editing, PCderived DAG synthesis, and finally the aggregation of these pathways. We also describe experimental techniques for determining the relative flux between alternative DAG synthesis pathways in vivo and the evidence for which plant species primarily utilize each DAG synthesis pathway. In particular, there is strong evidence that some plants utilize PC-derived DAG to generate TAG laden with PUFA, and that some plants primarily utilize de novo DAG to generate TAG rich in 18:1. However, uncertainty in the relative flux between DAG/TAG synthesis pathways still remains in most plants that accumulate unusual FA produced on PC. Finally, we discuss how different DAG synthesis pathways can cause bottlenecks in engineering of TAG FA 


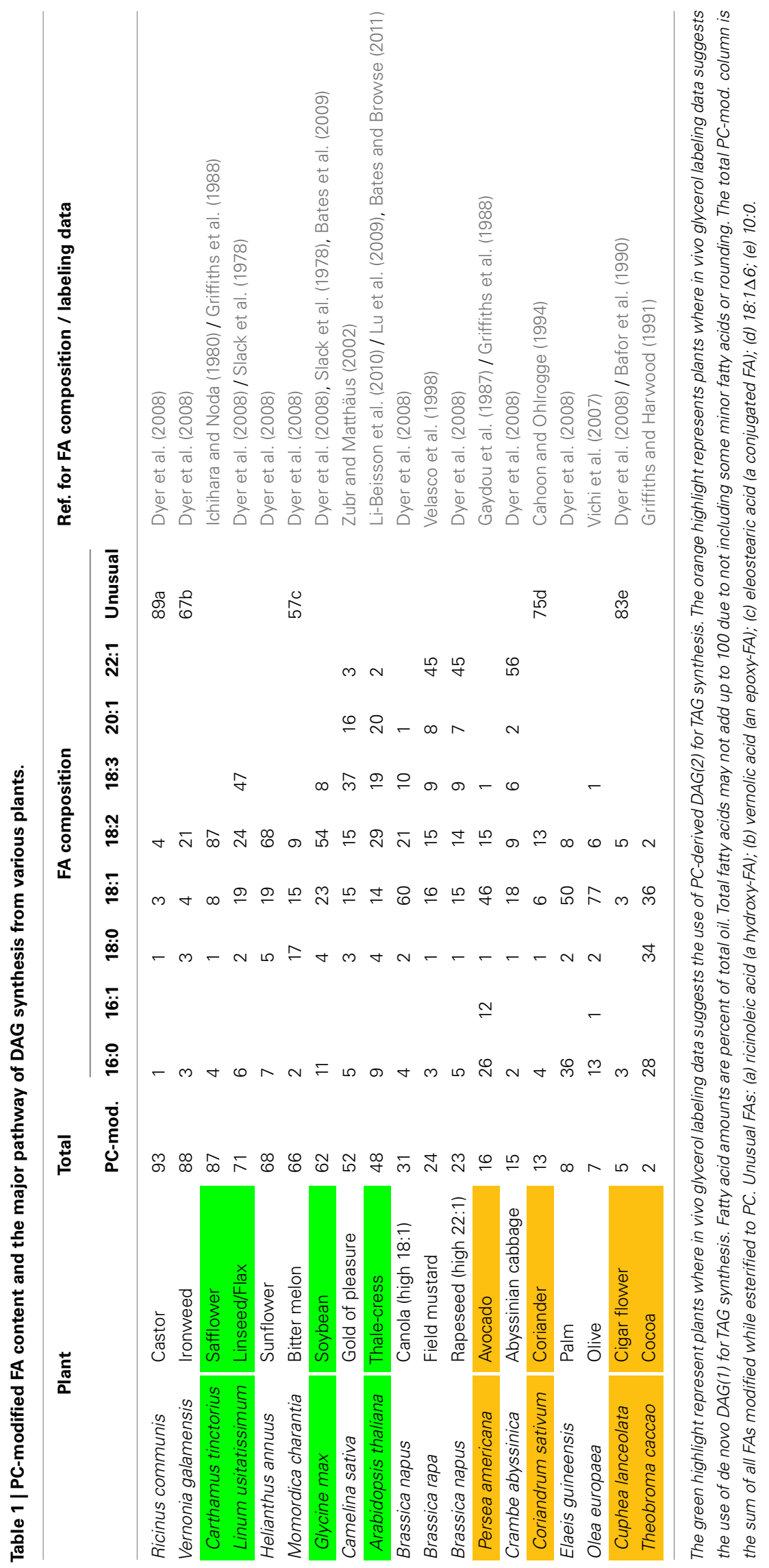


composition and potential strategies to overcome the bottlenecks in oilseed engineering to generate crops with designer vegetable oil compositions.

\section{PATHWAYS TO PRODUCE DAG/TAG WITH DIFFERENT FA COMPOSITIONS \\ DE NOVO DAG/TAG SYNTHESIS (KENNEDY PATHWAY)}

The simplest way to produce DAG and subsequent TAG is to utilize the products of FA synthesis exported from the plastid directly for de novo DAG/TAG synthesis (Figure 1). FA synthesis up to 18 carbons and one double bond takes place within the plastid (Li-Beisson et al., 2010). In the majority of plants the FAs 18:1 $\Delta 9>>16: 0>18: 0$ are the major FA products exported from the plastid for glycerolipid synthesis in the endoplasmic reticulum (ER). Upon exiting the plastid newly synthesized FAs are esterified to Coenzyme A (CoA) and the corresponding fatty acyl-CoA can be utilized for glycerolipid synthesis by sequential esterification to the $s n-1$ and $s n-2$ positions of glycerol-3-phosphate producing lyso-phosphatidic acid and subsequently phosphatidic acid by the acyl-CoA:glycerol-3-phosphate acyltransferase and acyl-CoA:lyso-phosphatidic acid acyltransferase, respectively. The ensuing phosphate removal by phosphatidic acid phosphatase produces de novo DAG (DAG(1), Figure 1). De novo DAG(1) can be utilized for TAG synthesis by acyl-CoA:diacylglycerol acyltransfease (DGAT; Figure 1; Li-Beisson et al., 2010). The three sequential acylations of glycerol-3-phosphate with acyl-CoA to produce de novo DAG(1) and then TAG is also referred to as the Kennedy pathway (Weiss and Kennedy, 1956; Weiss et al., 1960). De novo DAG(1)/TAG synthesis can also utilize plastid produced unusual FAs with shorter chain lengths, such as lauric acid (12:0) found in coconut (Cocos nucifera; Dyer et al., 2008), or unusual FAs with variations in double bond location, such as petroselinic acid (18:1 $\Delta 6)$ found in coriander (Coriandrum sativum; Cahoon and Ohlrogge, 1994). Additionally, the de novo
DAG(1)/TAG synthesis pathway can utilize acyl-CoA elongated to $\geq 20$ carbons in the cytosol and ER (Figure 1), such as erucic acid $(22: 1 \Delta 13)$ found in rapeseed (Brassica napus; Dyer et al., 2008).

\section{ACYL EDITING CAN PROVIDE PC-MODIFIED FA FOR DE NOVO DAG/TAG SYNTHESIS}

The vast majority of oilseeds rely on enzymes that desaturate or otherwise modify FAs esterified to the membrane lipid PC to produce TAG containing a diverse FA composition. For example, the common PC-modified FAs found in membrane and storage lipids, 18:2 and 18:3, are produced by ER localized desaturases that utilize PC as a substrate (Stymne and Stobart, 1987; Sperling et al., 1993). 18:1-PC is desaturated to $18: 2$ and then $18: 3$ by the FAD2 (Okuley et al., 1994) and FAD3 (Arondel et al., 1992) enzymes, respectively. Additionally, PC is the site for production of many uncommon FAs with unusual functional groups or double bond positions. For example, ricinoleic acid (12-hydroxy-9-cis-octadecenoic acid) is produced from 18:1-PC in castor (Ricinus communis) by a FA hydroxylase (Moreau and Stumpf, 1981; Bafor et al., 1991; Vandeloo et al., 1995). To incorporate PC-modified FAs into vegetable oils requires coordination of TAG synthesis with the flux of FAs into and out of PC.

Newly synthesized 18:1-CoA can be incorporated into PC for FA modification by the synthesis of PC from de novo DAG(1), or 18:1 can be directly incorporated into PC through a process known as "acyl editing" (Figure 2; Bates et al., 2007; Bates et al., 2009; Li-Beisson et al., 2010). Acyl editing is a deacylation-reacylation cycle that does not lead to net PC synthesis. The cycle starts with the release of an acyl group from PC, generating lyso-PC by the reverse action of acyl-CoA:lyso-phosphatidylcholine acyltransferase (LPCAT; Stymne and Stobart, 1984) or a phospholipase A (Chen et al., 2011). Re-esterification of lyso-PC by LPCAT generates $\mathrm{PC}$ and completes the cycle (Figure 2). Through acyl editing

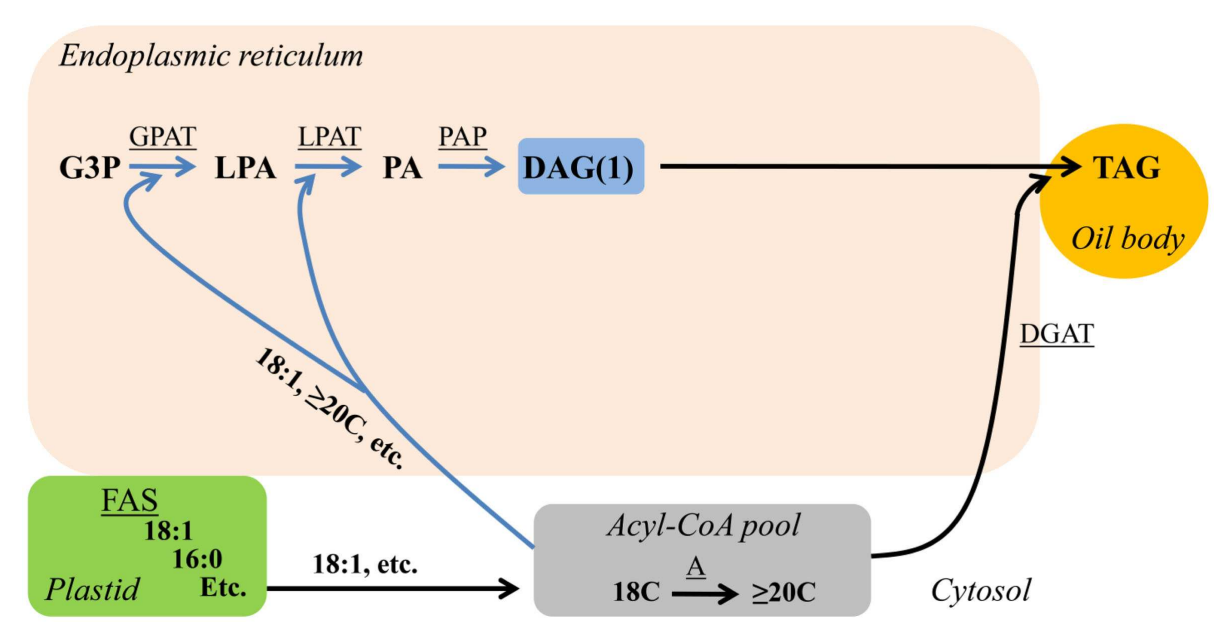

FIGURE 1 | De novo DAG/TAG synthesis (Kennedy pathway). Blue arrows indicate reactions involved in de novo DAG(1) synthesis. Substrate abbreviations: DAG, diacylglycerol; G3P, glycerol-3-phosphate; LPA, lyso-phosphatidic acid; PA, phosphatidic acid; PC, phosphatidylcholine;
TAG, triacylglycerol. Enzymatic reactions are underlined: A, FA elongation; DGAT, acyl-CoA:DAG acyltransferase; FAS, fatty acid synthesis; GPAT, acyl-CoA:G3P acyltransferase; LPAT, acyl-CoA:LPA acyltransferase; PAP, PA phosphatase. 


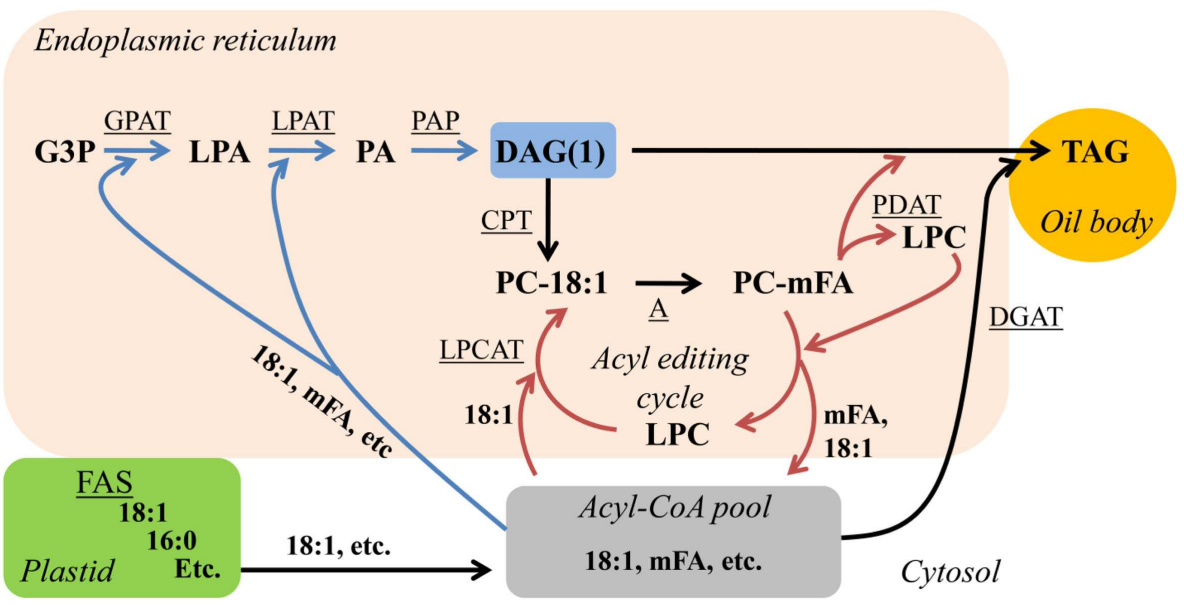

FIGURE 2 | Acyl editing can provide PC-modified FAs for de novo DAG/TAG synthesis. Blue arrows indicate reactions involved in de novo DAG(1) synthesis. Red arrows indicate reactions involved in acyl editing. Substrate abbreviations: DAG, diacylglycerol; G3P, glycerol-3-phosphate; LPA, lyso-phosphatidic acid; LPC, lyso-phosphatidylcholine; mFA, PC-modified FA; PA, phosphatidic acid; PC, phosphatidylcholine; TAG, triacylglycerol. Enzymatic reactions are underlined: $A$, modification of FA esterified to PC; CPT, CDP-choline:DAG cholinephosphotransferase; DGAT, acyl-CoA:DAG acyltransferase; FAS, fatty acid synthesis; GPAT, acyl-CoA:G3P acyltransferase; LPAT, acyl-CoA:LPA acyltransferase; LPCAT, acyl-CoA:LPC acyltransferase; PAP, PA phosphatase; PDAT, phospholipid:DAG acyltransferase. newly synthesized 18:1-CoA is channeled into PC for modification and the corresponding modified FAs can enter the acyl-CoA pool to be utilized for production of de novo DAG(1) containing PC-modified FAs (Stymne and Stobart, 1987; Bafor et al., 1991; Bates et al., 2007; Bates et al., 2009). However, not all FAs that enter the acyl editing cycle are destined for modification on PC. In coriander most 18:1 $\Delta 6$ exported from the plastid fluxes through PC prior to incorporation into de novo DAG/TAG (Cahoon and Ohlrogge, 1994). Recently it has been suggested that lyso-PC as part of the acyl editing cycle may be the major acceptor of nascent FA as they exit the plastid (Tjellström et al., 2012). Thus in many cases nascent FAs may flux through PC and back into the acyl-CoA pool (by the acyl editing cycle), regardless of whether the FA will be further modified on PC or not (Figure 2).

An additional aspect of acyl editing is the direct transfer of a FA from PC to DAG producing TAG by the phospholipid:diacylglycerol acyltransferase (PDAT; Dahlqvist et al., 2000). PDAT transfers an acyl group from the $s n-2$ position of PC to the $s n-3$ hydroxyl of DAG generating TAG and also lyso-PC that can be reacylated to PC by LPCAT (Xu et al., 2012) through the acyl editing cycle (Figure 2). Therefore, the FA composition of TAG produced through a de novo DAG(1)/TAG pathway depends on the relative fluxes of FA export from the plastid, FA elongation, acyl editing, and modification of FAs on PC to produce a diverse acyl-CoA pool for de novo DAG(1) synthesis and on the relative flux of TAG synthesis through DGAT or PDAT (Figure 2).

\section{PC-DERIVED DAG/TAG SYNTHESIS}

A DAG pool rich in PC-modified FAs for TAG assembly can also be produced through PC-derived DAG synthesis (Figure 3). The pathway of PC-derived DAG(2) synthesis starts with production of PC from de novo DAG(1) by CDP-choline:diacylglycerol cholinephosphotransferase (CPT; Li-Beisson et al., 2010) or phosphatidylcholine:diacylglycerol cholinephosphotransferase (PDCT; Lu et al., 2009; Figure 3). PC synthesis through CPT leads to a net production of PC from DAG. However, PDCT activity transfers the phosphocholine headgroup from PC to DAG generating new molecular species of DAG and PC, and thus does not lead to net accumulation of PC or DAG. Once PC is formed from de novo DAG(1) by either CPT or PDCT, the FAs esterified to PC are available for FA modification and acyl editing (Figure 2) to generate new molecular species of PC. The DAG substrate for TAG synthesis (DAG(2), Figure 3) is subsequently derived from PC by removal of the phosphocholine headgroup. PC-derived DAG(2) can be produced at the same time as PC production from de novo DAG(1) by the symmetrical activity of PDCT (Figure 3; Lu et al., 2009). The interconversion of the PC and DAG pools by PDCT is thus a mechanism to efficiently channel de novo DAG(1) into PC for further FA modification, and to liberate PC-derived DAG(2) containing modified FAs from PC for TAG synthesis. Production of PC-derived DAG(2) has also been demonstrated by the reverse action of CPT (Slack et al., 1983; Slack et al., 1985). Lipase mediated pathways utilizing phospholipase $\mathrm{C}$, or phospholipase D (Lee et al., 2011) and phosphatidic acid phosphatase to release DAG from PC may also contribute to the PC-derived DAG(2) pool. Either DGAT or PDAT may utilize PC-derived DAG(2) for the final TAG production.

PC-derived DAG(2) synthesis requires production of DAG twice: De novo DAG(1) synthesis, subsequent conversion to PC, and then PC-derived DAG(2) synthesis (Figure 3). Since PC is the site of FA modification the molecular species of PC-derived DAG(2) are typically significantly different than the molecular species of de novo DAG(1) utilized to produce PC. PC-derived DAG(2) synthesis may primarily be a mechanism to produce a DAG substrate for TAG synthesis with an increased content of 


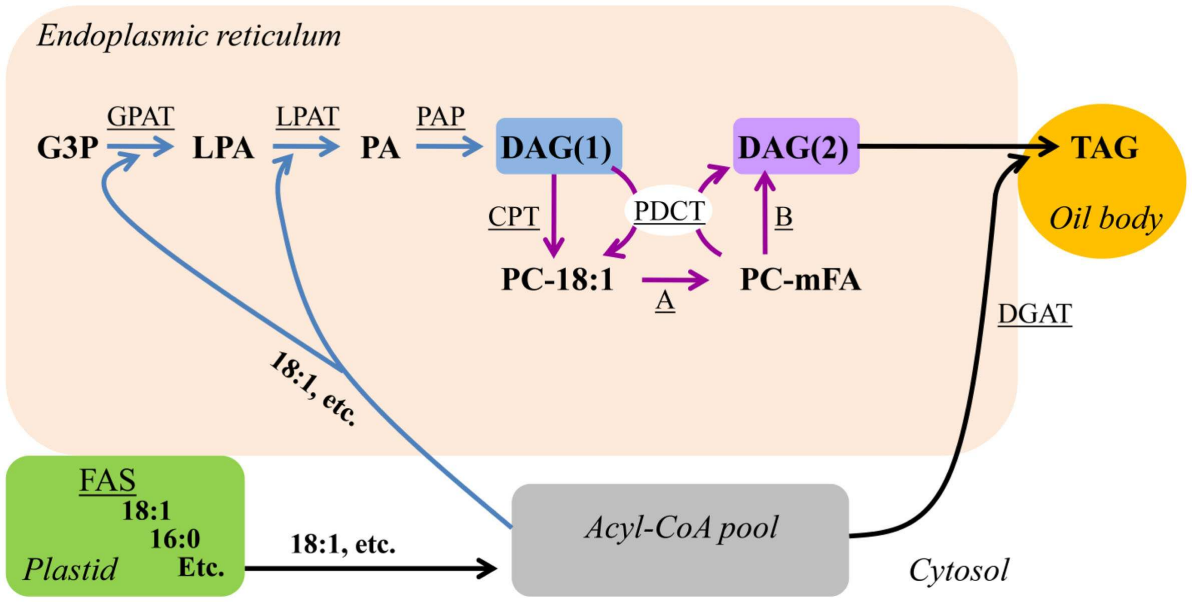

FIGURE 3 | PC-derived DAG/TAG synthesis. Blue arrows indicate reactions involved in de novo DAG(1) synthesis. Purple arrows indicate reactions involved in PC-derived DAG(2) synthesis. PC-derived DAG/TAG synthesis may also utilize acyl editing as in Figure 4. Substrate abbreviations: DAG, diacylglycerol; G3P, glycerol-3-phosphate; LPA, lyso-phosphatidic acid; mFA, PC-modified FA; PA, phosphatidic acid; PC, phosphatidylcholine; TAG, triacylglycerol. Enzymatic reactions are underlined: $A$, modification of FA esterified to $\mathrm{PC}$; $\mathrm{B}$, reversible CPT or phospholipase $\mathrm{C}$ or phospholipase D/PAP DAG production. CPT, CDP-choline:DAG cholinephosphotransferase; DGAT, acyl-CoA:DAG acyltransferase; FAS, fatty acid synthesis; GPAT, acyl-CoA:G3P acyltransferase; LPAT, acyl-CoA:LPA acyltransferase; PAP, PA phosphatase; PDCT, PC:DAG cholinephosphotransferase.
PC-modified FA above what can be produced by acyl editing and de novo DAG(1) synthesis alone (Figure 2).

\section{PLANTS CONTAIN THE POSSIBILITY OF UTILIZING MULTIPLE PATHWAYS TO GENERATE DAG/TAG}

Enzymatic activities involved in de novo DAG(1) synthesis, acyl editing, and PC-derived DAG(2) synthesis have been found in microsomes of oil-accumulating tissues from many plants (Stymne and Stobart, 1987). As far as we know, all plants have the capability to utilize each of these mechanisms to produce TAG with various FA compositions (Figure 4). However, the relative flux of TAG synthesis from de novo DAG(1) or PC-derived DAG(2) in vivo during TAG synthesis can vary widely depending on plant species and ultimately affect the final oil composition. Additionally, the relative flux through each of these DAG/TAG synthesis pathways may vary between different tissues, developmental stages or environmental conditions to produce different TAG molecular species. Experimental techniques that allow the relative utilization of each DAG synthesis pathway for TAG synthesis to be determined are the subject of Section "Analysis of the Relative Flux through Alternative DAG Synthesis Pathways." The elucidation of the relative fluxes through different pathways of DAG/TAG synthesis may be crucial to understanding how plants produce TAG with different FA compositions and how we can engineer designer vegetable oils.

\section{ANALYSIS OF THE RELATIVE FLUX THROUGH ALTERNATIVE DAG SYNTHESIS PATHWAYS}

The relative fluxes through alternative metabolic pathways are elucidated through in vivo radioactive (or stable isotope) labeling experiments that follow the kinetics of a labeled substrate as it is metabolized from initial pathway precursors, through intermediate pools, to final pathway products. These techniques by themselves or combined with mutant analysis have been crucial to the elucidation of plant lipid biosynthetic pathways (Roughan and Slack, 1982; Stymne and Stobart, 1987; Harwood, 1996; Ohlrogge and Jaworski, 1997). Useful substrates for following glycerolipid metabolism are $\left[{ }^{3} \mathrm{H}\right]$ - or $\left[{ }^{14} \mathrm{C}\right]$-glycerol and $\left[{ }^{14} \mathrm{C}\right]$ acetate. Labeled glycerol incorporated into plant tissue is phosphorylated and enters glycerolipid metabolism through glycerol-3-phosphate acylation. The $\left[{ }^{14} \mathrm{C}\right]$ acetate is activated to acetyl-CoA and is utilized for FA synthesis within the plastid to produce newly synthesized $\left[{ }^{14} \mathrm{C}\right] \mathrm{FAs}$, or for elongation of cytosolic acyl-CoA. Glycerol labeling and acetate labeling are complementary, not interchangeable. Labeled glycerol is only incorporated into TAG through de novo DAG(1) synthesis and then either converted directly to TAG or to PC for PC-derived DAG(2)/TAG synthesis (Figure 4, red lines). However, the nascent $\left[{ }^{14} \mathrm{C}\right] \mathrm{FAs}$ can initially enter glycerolipids through de novo DAG(1) synthesis, acyl editing, or DGAT to produce $s n-3$ labeled TAG (Figure 4, blue lines). Glycerol labeling is particularly useful for determining the relative fluxes of TAG synthesis from de novo DAG(1) or PC-derived DAG(2) and $\left[{ }^{14} \mathrm{C}\right]$ acetate is particularly useful for measuring the relative rates of de novo DAG(1) synthesis and acyl editing.

Metabolic labeling experiments can be done continuously over a time-course or in a pulse-chase format. In a continuous metabolic labeling experiment, the relative time the labeled substrate takes to accumulate linearly in each metabolite of a pathway corresponds with the complete filling of the precursor pools to that metabolite. Thus, the metabolite with the shortest lag time is the initial pathway precursor and the metabolite with the longest lag time is the final pathway product (Segel, 1976; Ratcliffe and Shachar-Hill, 2006). In a pulse-chase metabolic labeling experiment, the tissue is labeled for a short period of time (the pulse), unincorporated labeled substrate 


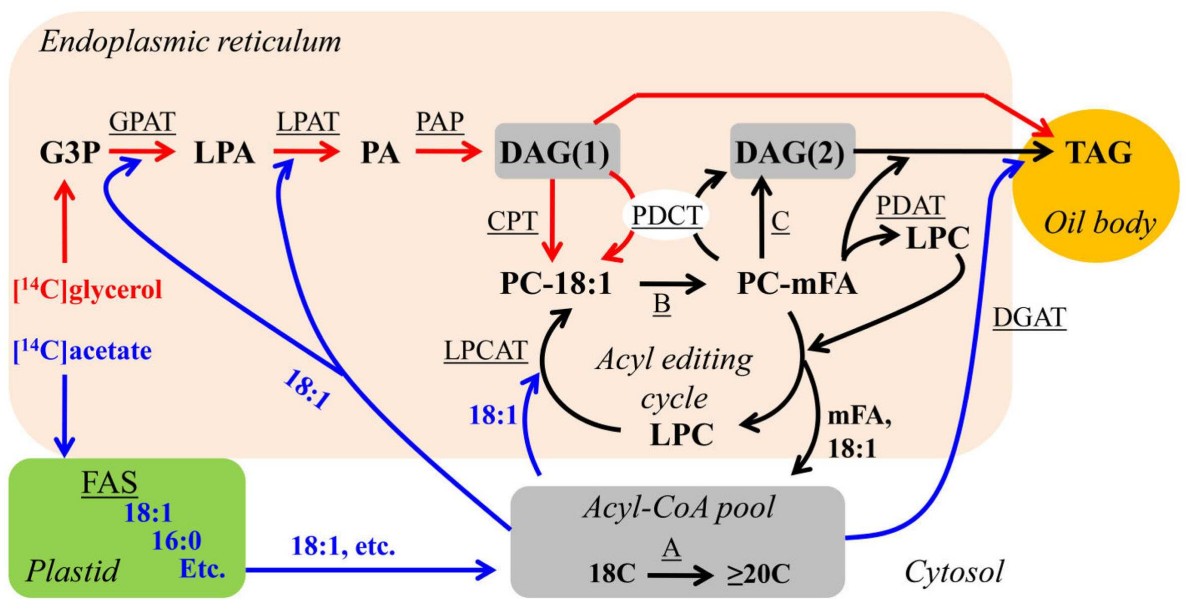

FIGURE 4 | Combined DAG/TAG synthesis pathways and initial labeling fluxes. Red arrows indicate possible initial fluxes of $\left[{ }^{14} \mathrm{C}\right.$ ]glycerol-labeled metabolites. Blue arrows indicate possible initial fluxes of $\left[{ }^{14} \mathrm{C}\right]$ acetate-labeled metabolites. Black arrows represent fluxes that will not be initially labeled with either $\left[{ }^{14} \mathrm{C}\right]$ glycerol or $\left[{ }^{14} \mathrm{C}\right]$ acetate, but will accumulate labeled metabolites over time. Substrate abbreviations: DAG, diacylglycerol; G3P, glycerol-3-phosphate; LPA, lyso-phosphatidic acid; mFA, PC-modified FA; PA, phosphatidic acid; PC, phosphatidylcholine:
TAG, triacylglycerol. Enzymatic reactions are underlined: A, FA elongation; $B$, modification of FA esterified to $\mathrm{PC}$; $C$, reversible $C P T$ or phospholipase C or phospholipase D/PAP DAG production. CPT, CDP-choline:DAG cholinephosphotransferase; DGAT, acyl-CoA:DAG acyltransferase; FAS, fatty acid synthesis; GPAT, acyl-CoA:G3P acyltransferase; LPAT, acyl-CoA:LPA acyltransferase; LPCAT, acyl-CoA:LPC acyltransferase; PAP, PA phosphatase; PDAT, phospholipid:DAG acyltransferase; PDCT, PC:DAG cholinephosphotransferase. is removed, and the tissue is further incubated with an unlabeled substrate over a time-course (the chase). Within a pulsechase experiment metabolites that lose label during the chase indicate the pathway precursors and metabolites that gain label indicate the end products (Ratcliffe and Shachar-Hill, 2006). In tissue that is actively synthesizing TAG as the major cellular glycerolipid, the relative incorporation of labeled glycerol from initially labeled de novo DAG(1) into the backbones of TAG and PC over time in a continuous labeling or during a pulsechase indicates the relative flux of TAG synthesis from de novo DAG(1) and PC-derived DAG(2), respectively (Figure 4, red lines).

Two key experimental details for determining relative flux through competing TAG synthesis pathways are: First, the use of tissue that is growing and rapidly accumulating TAG as similar to in planta as possible. Care should be taken to minimize changes in metabolism due to wounding, changes in osmotic potential, or incubation on unusual carbon sources. Tissue excised from plants should be collected at the peak of oil synthesis, utilized for labeling as soon as possible, and provided unlabeled carbon sources similar to the endogenous sources as well as the labeled substrate. Oilseed embryos grown in culture that accumulate TAG with similar quality and quantity to in planta have been very useful for this purpose (Allen et al., 2009; Bates et al., 2009). The use of plant derived suspension and callus cultures are a useful medium to rapidly apply radiolabels for kinetic studies of FA/membrane lipid synthesis. However, lipid class composition and TAG quantities of suspension/callus cultures are more similar to root or shoot tissue than oil bearing tissues, and thus these culture systems are less useful for deciphering the endogenous TAG biosynthetic pathways of oil bearing fruit or seeds (Williams et al., 1993; Tjellström et al., 2012). The second important factor to consider in labeling experiments is the optimum time-course that allows analysis of metabolite pools as they are accumulating label in a continuous labeling experiment or being depleted in label during a pulse-chase experiment. Once intermediate pools of a metabolic pathway reach saturation with label, the relative labeling between pathway intermediate metabolites reflects the pool size of each metabolite within the pathway and not the order of labeling. Therefore, a continuous labeling time-course with time points short enough so that the accumulation of label reflects the pathway order is required. Likewise, in a pulse-chase experiment the chase time points need to be long enough to measure the flux of label out of the pathway intermediates and into the products.

\section{LABELING EXPERIMENTS THAT INDICATE DIFFERENTIAL USE OF DAG SYNTHESIS PATHWAYS FOR TAG PRODUCTION IN DIFFERENT PLANTS THE USE OF PC-DERIVED DAG FOR TAG SYNTHESIS}

The utilization of PC-derived DAG(2) for TAG synthesis has been firmly established in several oilseed species that accumulate large amounts ( $\geq 48 \%$ ) of PC-modified FA (mostly PUFA) in TAG (Table 1). PUFA are produced on PC and naturally accumulate to high levels within membrane lipids of the ER, thus PC-derived DAG(2) provides an opportune source of DAG with a high PUFA content for TAG synthesis. Pulse-chase labeling of excised developing soybean (Glycine max) and linseed (Linum usitatissimum) cotyledons with $\left[{ }^{3} \mathrm{H}\right]$ glycerol demonstrated that the initially backbone labeled PC molecular species containing mostly 18:1 were converted to PUFA-containing DAG and TAG molecular species during the chase (Slack et al., 1978). This suggested a role for PC-derived DAG(2) in providing PUFA-rich molecular species of DAG for TAG synthesis. Time-course and pulse-chase 
$\left[{ }^{14} \mathrm{C}\right]$ glycerol labeling of developing safflower (Carthamus tinctorius) cotyledon slices (Griffiths et al., 1988) and multiple analyses of TAG synthesis in safflower microsomes (Stymne and Stobart, 1987) indicated that rapid interconversion of DAG(1) and PC with corresponding PC-FA modification can provide PUFAcontaining DAG(2) for TAG synthesis. A mutant screen in Arabidopsis (Arabidopsis thaliana) for changes in seed TAG PUFA content discovered a new enzyme, phosphatidylcholine:diacylglycerol cholinephosphotransferase (PDCT), which transfers the phosphocholine headgroup from PC to DAG, generating new molecular species of PC and DAG. Analysis of the pdct mutant (rodl) in Arabidopsis indicated that the PUFA content of pdct seeds was reduced $\sim 40 \%$ and pulse-chase labeling with $\left[{ }^{14} \mathrm{C}\right]$ glycerol indicated that PDCT is responsible for the majority of the PCDAG interconversion in developing Arabidopsis seeds (Lu et al., 2009).

Recently we re-investigated the flux through the competing pathways of de novo DAG(1)/TAG synthesis and PC-derived DAG(2)/TAG synthesis in developing soybeans to provide a more quantitative model of acyl fluxes through oilseed lipid metabolism (Bates et al., 2009). Cultured soybean embryos were utilized to minimize changes in metabolism due to wounding during tissue collection, and $\left[{ }^{14} \mathrm{C}\right]$ glycerol labeling with very short time points (e.g., $2 \mathrm{~min}$ ) allowed analysis of the initial products of glycerolipid synthesis (Figure 4, red lines). Molecular species analysis of initially labeled DAG indicated that de novo DAG(1) contained more 18:1 and less PUFA than bulk cellular DAG. Bulk DAG was much more similar to bulk PC in acyl composition than de novo DAG(1), consistent with bulk cellular DAG being derived from PC. Comparison of the initial rates of PC and TAG synthesis from $\left[{ }^{14} \mathrm{C}\right]$ glycerol-labeled de novo $\mathrm{DAG}(1)$ indicated that the major flux of de novo DAG(1) (>90\%) was utilized for PC synthesis (Figure 3), rather than directly contributing to TAG synthesis (Figure 1). In oilseeds, TAG accumulates to levels at least 20 -fold higher than membrane lipids, indicating over 95\% of total DAG synthesized will be utilized to produce TAG. Thus the very high initial rates of PC synthesis in developing soybeans actively accumulating TAG indicate the predominant pathway of TAG synthesis (>90\%) is through PC-derived DAG(2) (Figure 3).

Additionally, $\left[{ }^{14} \mathrm{C}\right]$ acetate labeling of the cultured soybean embryos was utilized to measure the flux of newly synthesized FAs into glycerolipids (Figure 4, blue lines; Bates et al., 2009). In these experiments, PC was labeled much more rapidly through the acyl editing cycle than synthesis of either de novo DAG(1) or TAG. Stereochemical analysis of nascent $\left[{ }^{14} \mathrm{C}\right] \mathrm{FA}$ incorporation into initially labeled DAG and TAG indicated that DAG was approximately equally labeled at the $s n-1$ and $s n-2$ positions while TAG was mostly labeled at the $s n-3$ position, further suggesting that very little or no TAG in soybeans is produced from initially synthesized de novo DAG(1). Molecular species analysis of $s n-3-$ $\left[{ }^{14} \mathrm{C}\right] \mathrm{FA}-\mathrm{TAG}$ indicated that the DAG molecular species utilized to synthesize TAG had a FA composition that was much more similar to bulk PC and bulk DAG than to de novo DAG(1), supporting the conclusions from the $\left[{ }^{14} \mathrm{C}\right]$ glycerol labeling that TAG is predominantly synthesized from PC-derived DAG(2) in soybeans. The initial rates of $\left[{ }^{14} \mathrm{C}\right]$ acetate labeling of $\mathrm{PC}>\mathrm{DAG} \sim \mathrm{TAG}$ combined with the $\left[{ }^{14} \mathrm{C}\right]$ glycerol labeling results suggested that both a high rate of acyl editing and the $>90 \%$ flux of TAG synthesis through PC-derived DAG(2) together (Figure 4) contribute to accumulate the high amount $(>60 \%)$ of PC-modified FA in TAG of soybeans (Table 1).

Very similar results to those of soybean were obtained with $\left[{ }^{14} \mathrm{C}\right]$ glycerol labeling of developing Arabidopsis seeds (Bates and Browse, 2011). $\left[{ }^{14} \mathrm{C}\right]$ glycerol-labeled de novo DAG(1) molecular species contained more 18:1 and less PUFA than bulk DAG, and bulk DAG FA composition was much more similar to bulk PC than de novo DAG(1). Additionally, the initial rates of glycerolipid synthesis from $\left[{ }^{14} \mathrm{C}\right]$ glycerol indicated that $>90 \%$ of $d e$ novo DAG(1) are initially utilized to synthesize PC during TAG synthesis (Bates and Browse, 2011), supporting and extending on the genetic evidence from the pdct mutant (Lu et al., 2009) that Arabidopsis primarily utilizes a PC-derived DAG(2) pathway (Figure 3) to accumulate high levels of PUFA in DAG for TAG synthesis. Recent characterization of an acyl editing cycle present in Arabidopsis cell cultures (Tjellström et al., 2012), combined with the fact that the pdct mutant still contains $\sim 60 \%$ of wild-type PUFA levels in TAG (Lu et al., 2009), together suggest that both PDCT mediated PC-derived DAG(2) synthesis and acyl editing contribute to production of PUFA-containing TAG in Arabidopsis (Figure 4).

\section{THE USE OF DE NOVO DAG FOR TAG SYNTHESIS}

In plants that accumulate TAG containing mostly the products of FA synthesis exported from the plastid (e.g., 18:1, 16:0, etc.) as opposed to PC-modified FAs, it appears that the direct utilization of de novo DAG(1) for TAG synthesis (or Kennedy pathway) is the major pathway of oil accumulation (Figure 1). Avocado (Persea americana) fruit mesocarp produces TAG that is rich in 18:1 and low in PC-modified FAs (Table 1). A time-course incorporation of $\left[{ }^{14} \mathrm{C}\right]$ glycerol in avocado labeled mostly intermediates of the Kennedy pathway and had very little PC labeling (Griffiths et al., 1988). Analogous results were also found with $\left[{ }^{3} \mathrm{H}\right]$ glycerol labeling of cocoa (Theobroma cacao) cotyledons, which accumulate TAG containing mostly 16:0, 18:0, and 18:1 (Griffiths and Harwood, 1991). A similar scenario may be true for plants that accumulate oil rich in unusual FA that are produced in the plastid as part of FA synthesis, while only trace amounts of these unusual FA accumulate in membrane lipids. For instance, Cuphea lanceolata accumulates $>80 \%$ of TAG FA as 10:0 and $<1 \%$ accumulates in PC. $\left[{ }^{14} \mathrm{C}\right]$ glycerol labeling of detached C. lanceolata cotyledons indicated very little $\mathrm{PC}$ synthesis during TAG accumulation. Assays of $C$. lanceolata microsomes suggest that enzymatic acyl selectivity may channel di-10:0-DAG into TAG synthesis and DAG containing 16- and 18-carbon FA into membrane lipid synthesis (Bafor et al., 1990 ). The $\leq 16 \%$ PC-modified FA that does accumulate in TAG of these species (Table 1) may enter the acyl-CoA pool through acyl editing and is utilized by de novo DAG(1)/TAG synthesis (Figure 2). For example, $\left[{ }^{14} \mathrm{C}\right]$ glycerol labeling indicated coriander endosperm primarily utilizes a de novo DAG/TAG pathway and coriander accumulates TAG containing 75\% 18:1 $\Delta 6$ (which is not modified on PC) and only 13\% PUFA (Table 1). However, $\left[{ }^{14} \mathrm{C}\right]$ acetate labeling indicated that most nascent $18: 1 \Delta 6$ exported from the plastid enter PC (by the acyl editing cycle), 
prior to de novo DAG(1)/TAG synthesis (Cahoon and Ohlrogge, 1994). Therefore, the acyl editing cycle can be a major flux within acyl lipid metabolism of oil bearing tissues even in species that do not accumulate large amounts of PC-modified FA in TAG.

\section{UNCERTAINTY IN THE DAG SYNTHESIS PATHWAY FOR PLANTS THAT SYNTHESIZE UNUSUAL FA ON PC}

The membrane lipid PC is the site for production of many modified FA with unusual functional groups (hydroxy, epoxy, etc.), or unusual double bond positions (e.g., conjugated) (Dyer et al., 2008). In most plant species that accumulate unusual PC-modified FAs in TAG very little of the PC-modified FAs accumulate in the membrane lipids (Stahl et al., 1995; Millar et al., 2000). The exact mechanisms that allow efficient channeling of unusual PCmodified FAs out of PC where they are synthesized and into TAG are unknown in most plants. In particular uncertainty lies in whether the PC-modified FAs are channeled into TAG through efficient acyl editing and de novo DAG(1)/TAG synthesis (Figure 2) or through PC-derived DAG(2)/TAG synthesis (Figure 3), or if both mechanisms (Figure 4) can contribute to the flux of unusual FAs out of PC and into TAG. For example, ricinoleic acid (12hydroxy-9-cis-octadecenoic acid) is produced from 18:1 at the $s n-2$ position of PC by the castor FA hydroxylase (Moreau and Stumpf, 1981; Bafor et al., 1991). Castor is one of many plants that produce hydroxy-FA (HFA) on PC by a variant of the FAD2 enzyme (Badami and Patil, 1980; Vandeloo et al., 1995; Broun et al., 1998; Dauk et al., 2007). In castor endosperm HFA accumulates to $\sim 90 \%$ of the FA in TAG (Table 1 ; and over $70 \%$ of seed TAG contain three HFA; Lin et al., 2003). However during production of HFA-containing TAG, HFA only accumulates to $~ 5 \%$ of FA in PC (Stahl et al., 1995). This requires that at least 90\% of seed 18:1 fluxes into the $s n-2$ position of PC where it is hydroxylated, and then the HFA is efficiently removed from $\mathrm{PC}$ and redistributed into the $s n-1, s n-2$, and $s n-3$ positions of TAG through acyl editing and de novo DAG(1)/TAG synthesis, or PC-derived DAG(2)/TAG synthesis, or both.

Careful in vitro analysis of TAG synthesis in microsomes from castor endosperm suggests that ricinoleate is efficiently released from PC through acyl editing and the ricinoleoyl-CoA produced is efficiently utilized to synthesize de novo DAG(1) and TAG (Figure 2; Bafor et al., 1991). In castor microsomes di-HFA-DAG is more efficiently utilized for TAG synthesis than mono-HFA-DAG. However, PC synthesis does not appear to have a selectivity for or against any HFA-DAG molecular species (Vogel and Browse, 1996). Therefore, it is possible that de novo synthesized 1-HFA-218:1-sn-DAG(1) may be converted to PC for further hydroxylation and the corresponding di-HFA-PC converted to DAG(2) for TAG synthesis (Figure 4). Castor microsomes fed with $\left[{ }^{14} \mathrm{C}\right]$ glycerol3-phosphate and HFA-CoA do synthesize labeled PC at $\sim 1 / 3$ the rate of TAG synthesis, suggesting that both de novo DAG(1) and PC-derived DAG(2) may be able to contribute to TAG synthesis in vivo (Bafor et al., 1991).

Recently, a homolog of Arabidopsis PDCT with high activity was identified from a castor endosperm cDNA library. The castor PDCT enhanced the accumulation of HFA in TAG of transgenic plants co-expressing the castor FA hydroxylase, consistent with the ability of castor to utilize a PC-derived DAG(2) pathway of
TAG synthesis in vivo (Hu et al., 2012). Therefore, the symmetrical activity of PDCT may also be important for channeling de novo DAG(1) substrates into PC for unusual FA synthesis similar to its use in PUFA synthesis in Arabidopsis (Lu et al., 2009). Interestingly, a recent mining of the developing bitter melon (Momordica charantia) seed transcriptome failed to identify an expressed version of PDCT (Yang et al., 2010). Bitter melon accumulates high levels of unusual PC-modified FAs (conjugated FA, Table 1) in TAG. This result may suggest either that bitter melon may primarily utilize acyl editing and de novo DAG(1)/TAG synthesis to channel PC-modified FA from PC to TAG, or that bitter melon may contain a PC-derived DAG(2) flux that does not rely on a PDCT homologe. It is clear that different plants may use different DAG synthesis pathways and/or different enzymatic activities to channel unusual PC-modified FAs out of the membrane and into TAG. Additional in vivo metabolic labeling studies are needed in plants that accumulate unusual PC-modified FAs in TAG to determine the relative contribution of acyl editing combined with de novo DAG(1) synthesis and/or PC-derived DAG(2) synthesis (Figure 4) to TAG production in these species.

\section{EFFECT OF DAG SYNTHESIS PATHWAYS ON OILSEED ENGINEERING}

\section{PATHWAY BOTTLENECKS IN TRANSGENIC OILSEEDS}

Engineering a change in the relative abundance of FAs naturally occurring in seed oils has proved more straight forward than the accumulation of non-native unusual FAs in TAG of transgenic plants (Napier and Graham, 2010; Chapman and Ohlrogge, 2012). Introduction of transgenes that produce unusual modified FAs on PC typically cause higher accumulation of the modified FA in PC of the host plant than in the plant from which the transgene was obtained. Likewise, accumulation of the PC-modified FA in TAG is typically much less in the transgenic host than in the native source (Thomaeus et al., 2001; Cahoon et al., 2006; van Erp et al., 2011). In many cases transgenic production of PC-modified FAs also reduces total oil accumulation in the transgenic plant (Dauk et al., 2007; Bates and Browse, 2011; Li et al., 2012). These results indicate that many transgenic plants have biosynthetic bottlenecks between the site of modified FA production on PC and accumulation in TAG (Cahoon et al., 2007). Bottlenecks will be produced whenever the transgenic host plant does not efficiently utilize the unusual PC-modified FA by any part of the TAG biosynthetic pathway including, but not limited to: removal of modified FA from $\mathrm{PC}$, incorporation of the PC-modified FA into the acyl-CoA pool, de novo DAG(1) synthesis, PC and PC-derived DAG(2) synthesis, and final acylation of DAG by DGAT or PDAT.

We recently demonstrated that the large flux of TAG synthesis through PC-derived DAG(2) in Arabidopsis to be one such bottleneck in transgenic plants expressing the castor FA hydroxylase (Bates and Browse, 2011). $\left[{ }^{14} \mathrm{C}\right]$ glycerol labeling demonstrated acyl editing and de novo DAG(1) synthesis (Figure 2) could produce de novo DAG(1) containing HFA in Arabidopsis. However, HFA-containing de novo DAG(1) was not efficiently utilized directly for TAG synthesis (Figure 2) or incorporated into PC (Figure 3) and appeared to be turned over (Bates and Browse, 2011). Even though the flux of HFA-containing de novo DAG(1) into PC was restricted, the accumulation of HFA mostly at the 
$s n-2$ position of TAG was attributed to the substantial flux of HFA-containing PC-derived DAG(2) out of PC after production of HFA by the castor $s n-2-P C$ hydroxylase (Figure 3). Therefore, transgenic host plants that utilize predominantly a PC-derived DAG(2) pathway of TAG synthesis may be useful for accumulation of unusual PC-modified FAs at the $s n-2$ position of TAG. However, the bottleneck in conversion of de novo DAG(1) containing the unusual FA to PC may limit the accumulation of the unusual FA at the $s n-1$ position of TAG, and potentially lead to reduced total oil (Bates and Browse, 2011).

\section{POTENTIAL STRATEGIES TO OVERCOME THE BOTTLENECK IN UNUSUAL FA FLUX THROUGH THE PC-DERIVED DAG PATHWAY}

Plants that naturally accumulate unusual PC-modified FA, such as HFA in Castor, do not accumulate high levels of unusual PCmodified FAs in membrane lipids, even though they can be as high as $90 \%$ in TAG (Millar et al., 2000). Thus, these plants must contain mechanisms to avoid potential biosynthetic bottlenecks between membrane lipid and TAG synthesis, and efficiently transfer modified FAs from the site of synthesis on PC into TAG. In castor for instance, one such mechanism could be an efficient acyl editing cycle to channel HFA from PC into the Kennedy pathway (Figure 2; Bafor et al., 1991). Alternatively, if castor can also utilize the PC-derived DAG(2) pathway of TAG synthesis (Figure 3) it must allow HFA-containing de novo $\operatorname{DAG}(1)$ to be converted to PC, as suggested above by the lack of specificity for/against HFA-containing DAG molecular species by castor CPT (Vogel and Browse, 1996). The HFA-PC must then be efficiently converted to PC-derived DAG(2) to avoid HFA building up in the membrane. Efficient channeling of PC-modified FAs from PC to TAG may involve enzymatic specificities for unusual FAs or separation of the unusual FA based TAG synthesis, from membrane lipid synthesis in separate micro-domains of the ER (Shockey et al., 2006; Gidda et al., 2011). The co-expression of TAG synthesis enzymes (DGAT or PDAT) that are selective for PC-modified FAs together with PC-modified FA producing enzymes, has one strategy to enhance the flux of PC-modified FA into TAG of transgenic plants (Burgal et al., 2008; Li et al., 2010; Kim et al., 2011; van Erp et al., 2011; Li et al., 2012). The goal for overcoming the bottlenecks in transgenic plants must be to either both elucidate and replicate the TAG synthesis pathways of plants that naturally accumulate PC-modified FAs, or find alternative biosynthetic strategies to circumvent bottlenecks in oilseed engineering.

Engineering strategies to overcome the bottleneck in conversion of de novo DAG(1) containing unusual FA to PC in transgenic plants may involve co-expression of unusual-FA-specific PC-synthesizing enzymes (CPT or PDCT) with unusual FA synthesis enzymes. An alternative approach could be to redirect the flux of de novo DAG(1) from PC synthesis directly to TAG synthesis. Recent studies co-expressing the castor FA hydroxylase and castor PDCT in Arabidopsis has achieved increased accumulation of HFA in TAG and less in PC, as well as mostly relieving the HFA induced reduced oil phenotype (Hu et al., 2012). However, it is unknown if over-expression of castor PDCT increased the flux of HFA-containing de novo DAG(1) into PC or if the results were due to an increased flux of HFA-containing PC-derived DAG(2) out of PC, thereby reducing HFA acyl editing and synthesis of
HFA-containing de novo DAG(1). Expression of the castor FA hydroxylase in the Arabidopsis pdct mutant reduced HFA accumulation in TAG and total seed oil compared to expression of the FA hydroxylase in wild-type Arabidopsis (Hu et al., 2012). These results support the hypothesis based on the accumulation of HFA at the $s n-2$ position of TAG (Bates and Browse, 2011; van Erp et al., 2011) that the flux of HFA-containing PC-derived DAG(2) out of PC is important for HFA-TAG accumulation in transgenic Arabidopsis. Additionally, these results suggest that the loss of PDCT activity does not efficiently shift the flux of HFA from the PCderived DAG(2) pathway (Figure 3) to the acyl editing/de novo DAG(1) pathway (Figure 2) for accumulation in TAG.

\section{ALTERNATIVE HOST PLANT SPECIES MAY HOLD THE KEY TO ACCUMULATION OF UNUSUAL FA IN TRANSGENIC PLANTS}

An alternative bioengineering scenario for accumulation of unusual FAs in TAG of transgenic plants would be to utilize a host plant that does not rely on a PC-derived DAG(2) pathway of TAG synthesis. It is possible a de novo DAG(1)/TAG synthesis pathway may be more able to accommodate unusual FAs since de novo DAG(1) containing unusual FAs will be directly available for TAG synthesis and less likely to affect membrane lipid FA composition. Plants identified thus far that primarily utilize a de novo DAG(1)/TAG biosynthetic pathway accumulate mostly FAs that are not modified on PC (Table 1). Therefore, removal of unusual PC-modified FAs from PC into the acyl-CoA pool could potentially represent a bottleneck in these plants. Identifying, or engineering, a suitable crop plant that has a high rate of acyl editing to remove unusual PC-modified FAs from PC and also primarily utilizes a de novo DAG(1) pathway of TAG synthesis (Figure 2) may be ideal for engineering of designer oils containing unusual PC-modified FAs.

The relative fluxes of TAG synthesis from de novo DAG(1) or PC-derived DAG(2) are unknown in most plant species, especially those plant species that accumulate oils containing unusual PC-modified FAs. However, of the plant species where labeling data has indicated the relative flux between these pathways there appears to be a correlation between high PUFA content $(\geq 48 \%)$ in oil with a predominantly PC-derived DAG(2)/TAG synthesis pathway, and low PUFA content $(\leq 16 \%)$ in oil with a predominantly de novo DAG(1)/TAG synthesis pathway (Table 1). For plant species that accumulate intermediate amounts of PUFA $(20-40 \%)$ in oil it is uncertain if this level of PUFA accumulation is due to acyl editing and de novo DAG(1)/TAG synthesis alone, or if a mixture of both de novo DAG(1) and PC-derived DAG(2) provides substrate for TAG synthesis. Some plants in the family Brassicaceae have been suggested as potential species for production of designer oils because they are related to Arabidopsis for genetic resources and represent non-food oilseed crops. Based on PC-modified FA content (Table 1) we might expect Camelina sativa to have a similar PC-derived DAG(2)/TAG synthesis pathway as Arabidopsis. However, Crambe abyssinica which has similar PC-modified FA content to avocado may have a larger contribution of a traditional Kennedy pathway to TAG synthesis. Interestingly, the Arabidopsis pdct mutant has significantly reduced flux through PC-derived DAG(2) and still contains 29\% PCmodified FAs in TAG (Lu et al., 2009). This level of PC-modified 
FA accumulation in TAG may represent what Brassicaceae species can achieve through mostly acyl editing and de novo DAG(1)/TAG synthesis (Figure 2). Plants with similar endogenous levels of PCmodified FA to Arabidopsis pdct include B. napus, both high 22:1 containing wild-type rapeseed and the high 18:1/low 22:1 containing Canola (Table 1). Further research is needed to test the pathway of DAG/TAG synthesis in these and other species, and to determine the general extent of which PC-modified FA content in plant oils corresponds to the relative fluxes of TAG synthesis through de novo DAG(1) (Figures 1, 2) or PC-derived DAG(2) (Figure 3).

\section{CLOSING REMARKS}

It is clear that the pathway of DAG and subsequently TAG synthesis differs depending on plant species (Table 1) and can greatly affect the final oil composition. The utilization of PC-derived DAG(2) for TAG synthesis (Figure 3) is a major pathway of oil synthesis in many plant species, and appears to be a mechanism to enhance the PUFA content (or possibly other PC-derived PCmodified FAs) in the DAG pool above what accumulates through

\section{REFERENCES}

Allen, D. K., Ohlrogge, J. B., and Shachar-Hill, Y. (2009). The role of light in soybean seed filling metabolism. Plant J. 58, 220-234.

Arondel, V., Lemieux, B., Hwang, I., Gibson, S., Goodman, H. M., and Somerville, C. R. (1992). Mapbased cloning of a gene controlling omega-3-fatty-acid desaturation in Arabidopsis. Science 258, 1353-1355.

Badami, R. C., and Patil, K. B. (1980). Structure and occurrence of unusual fatty acids in minor seed oils. Prog. Lipid Res. 19, 119-153.

Bafor, M., Jonsson, L., Stobart, A. K., and Stymne, S. (1990). Regulation of triacylglycerol biosynthesis in embryos and microsomal preparations from the developing seeds of Cuphea lanceolata. Biochem. J. 272, 31-38.

Bafor, M., Smith, M. A., Jonsson, L., Stobart, K., and Stymne, S. (1991). Ricinoleic acid biosynthesis and triacylglycerol assembly in microsomal preparations from developing castor-bean (Ricinus communis) endosperm. Biochem. J. 280, 507-514.

Bates, P. D., and Browse, J. (2011). The pathway of triacylglycerol synthesis through phosphatidylcholine in Arabidopsis produces a bottleneck for the accumulation of unusual fatty acids in transgenic seeds. Plant J. 68, 387-399.

Bates, P. D., Durrett, T. P., Ohlrogge, J. B., and Pollard, M. (2009). Analysis of acyl fluxes through multiple pathways of triacylglycerol synthesis in developing soybean embryos. Plant Physiol. 150, 55-72.

Bates, P. D., Ohlrogge, J. B., and Pollard, M. (2007). Incorporation of newly synthesized fatty acids into cytosolic glycerolipids in pea leaves occurs via acyl editing. J. Biol. Chem. 282, 31206-31216.

Baud, S., and Lepiniec, L. (2010). Physiological and developmental regulation of seed oil production. Prog. Lipid Res. 49, 235-249.

Broun, P., Boddupalli, S., and Somerville, C. (1998). A bifunctional oleate 12-hydroxylase: desaturase from Lesquerella fendleri. Plant J.13, 201-210.

Burgal, J., Shockey, J., Lu, C. F., Dyer, J., Larson, T., Graham, I., and Browse, J. (2008). Metabolic engineering of hydroxy fatty acid production in plants: RcDGAT2 drives dramatic increases in ricinoleate levels in seed oil. Plant Biotechnol. J. 6, 819-831.

Cahoon, E. B., Dietrich, C. R., Meyer, K., Damude, H. G., Dyer, J. M., and Kinney, A. J. (2006). Conjugated fatty acids accumulate to high levels in phospholipids of metabolically engineered soybean and Arabidopsis seeds. Phytochemistry 67, 1166-1176.

Cahoon, E. B., and Ohlrogge, J. B. (1994). Apparent role of phosphatidylcholine in the metabolism of petroselinic acid in developing umbelliferae endosperm. Plant Physiol. 104, 845-855.

Cahoon, E. B., Shockey, J. M., Dietrich, C. R., Gidda, S. K., Mullen, R. T., and Dyer, J. M. (2007). Engineering oilseeds for sustainable production

acyl editing and de novo DAG(1) synthesis alone (Figure 2). However, the synthesis of PC from de novo DAG(1) for eventual PC-derived DAG(2)/TAG synthesis can represent a bottleneck for engineering of some unusual FAs into oilseed plants (Bates and Browse, 2011). Deciphering the relative fluxes through alternative TAG synthesis pathways in different vegetable oil crops will be fundamental to our understanding of TAG synthesis in different plants. Additionally, the utilization of in vivo metabolic flux analysis to understand metabolic changes in transgenic crops may be crucial to our ability to identify biosynthetic bottlenecks, and design engineering strategies that overcome the bottlenecks for production of plant oils with designer FA compositions.

\section{ACKNOWLEDGMENTS}

We would like to thank Dr. John Ohlrogge for critically reading the manuscript and helpful suggestions. This work was supported by the U.S. National Science Foundation (grant no. DBI-0701919) and by the Agricultural Research Center at Washington State University.

of industrial and nutritional feedstocks: solving bottlenecks in fatty acid flux. Curr. Opin. Plant Biol. 10, 236-244.

Chapman, K. D., and Ohlrogge, J. B. (2012). Compartmentation of triacylglycerol accumulation in plants. J. Biol. Chem. 287, 2288-2294.

Chen, G., Snyder, C. L., Greer, M. S., and Weselake, R. J. (2011). Biology and biochemistry of plant phospholipases. CRC Crit. Rev. Plant Sci. 30, 239-258.

Dahlqvist, A., Stahl, U., Lenman, M., Banas, A., Lee, M., Sandager, L., Ronne, H., and Stymne, H. (2000). Phospholipid: diacylglycerol acyltransferase: An enzyme that catalyzes the acyl-CoA-independent formation of triacylglycerol in yeast and plants. Proc. Natl. Acad. Sci. U.S.A. 97, 6487-6492.

Dauk, M., Lam, P., Kunst, L., and Smith, M. A. (2007). A FAD2 homologue from Lesquerella lindheimeri has predominantly fatty acid hydroxylase activity. Plant Sci. 173, 43-49.

Dyer, J. M., Stymne, S., Green, A. G., and Carlsson, A. S. (2008). High-value oils from plants. Plant J. 54, 640-655.

Gaydou, E. M., Lozano, Y., and Ratovohery, J. (1987). Triglyceride and fatty acid compositions in the mesocarp of Persea americana during fruit development. Phytochemistry 26, 1595-1597.

Gidda, S. K., Shockey, J. M., Falcone, M. Kim, P. K., Rothstein, S. J., Andrews, D. W., Dyer, J. M., and Mullen, R. T. (2011). Hydrophobic-domaindependent protein-protein interactions mediate the localization of
GPAT enzymes to ER subdomains. Traffic 12, 452-472.

Griffiths, G., and Harwood, J. L. (1991). The regulation of triacylglycerol biosynthesis in cocoa (Theobroma cacao) L. Planta 184, 279-284.

Griffiths, G., Stymne, S., and Stobart, A. K. (1988). Phosphatidylcholine and its relationship to triacylglycerol biosynthesis in oil-tissues. Phytochemistry 27, 2089-2093.

Harwood, J. L. (1996). Recent advances in the biosynthesis of plant fatty acids. Biochim. Biophys. Acta Lipids Lipid Metab. 1301, 7-56.

$\mathrm{Hu}, \mathrm{Z}$., Ren, Z., and Lu, C. (2012). The phosphatidylcholine diacylglycerol cholinephosphotransferase is required for efficient hydroxy fatty acid accumulation in transgenic Arabidopsis. Plant Physiol. 158, 1944-1954.

Ichihara, K.-I., and Noda, M. (1980). Fatty acid composition and lipid synthesis in developing safflower seeds. Phytochemistry 19, 49-54.

Kim, H. U., Lee, K.-R., Go, Y. S., Jung, J. H., Suh, M.-C., and Kim, J. B. (2011). Endoplasmic reticulumlocated PDAT1-2 from castor bean enhances hydroxy fatty acid accumulation in transgenic plants. Plant Cell Physiol. 52, 983-993.

Knutzon, D. S., Hayes, T. R., Wyrick, A., Xiong, H., Maelor Davies, H., and Voelker, T. A. (1999). Lysophosphatidic acid acyltransferase from coconut endosperm mediates the insertion of laurate at the sn-2 position of triacylglycerols in lauric rapeseed oil and can increase total laurate levels. Plant Physiol. 120, 739-746. 
Lee, J., Welti, R., Schapaugh, W. T., and Trick, H. N. (2011). Phospholipid and triacylglycerol profiles modified by PLD suppression in soybean seed. Plant Biotechnol. J. 9, 359-372.

Li-Beisson, Y., Shorrosh, B., Beisson, F., Andersson, M., Arondel, V., Bates, P., Baud, S., Bird, D., Debono, A., Durrett, T., Franke, R., Graham, I., Katayama, K., Kelly, A., Larson, T., Markham, J., Miquel, M., Molina, I., Nishida, I., Rowland, O., Samuels, L., Schmid, K., Wada, H., Welti, R., $\mathrm{Xu}, \mathrm{C}$., Zallot, R., and Ohlrogge, J. (2010). Acyl lipid metabolism. Arabidopsis Book 8, 1-65.

Li, R., Yu, K., Hatanaka, T., and Hildebrand, D. F. (2010). Vernonia DGATs increase accumulation of epoxy fatty acids in oil. Plant Biotechnol. J. 8, 184-195.

Li, R., Yu, K., Wu, Y., Tateno, M., Hatanaka, T., and Hildebrand, D. F. (2012). Vernonia DGATs can complement the disrupted oil and protein metabolism in epoxygenaseexpressing soybean seeds. Metab. Eng. 14, 29-38.

Lin, J. -T., Turner, C., Liao, L. P., and Mckeon, T. A. (2003). Identification and quantification of the molecular species of acylglycerols in castor oil by HPLC using ELSD. J. Liq. Chromatogr. Relat. Technol. 26, 773-780.

Lu, C., Xin, Z., Ren, Z., Miquel, M., and Browse, J. (2009). An enzyme regulating triacylglycerol composition is encoded by the ROD1 gene of Arabidopsis. Proc. Natl. Acad. Sci. U.S.A. 106, 18837-18842.

Lu, C. F., Napier, J. A., Clemente, T. E., and Cahoon, E. B. (2011). New frontiers in oilseed biotechnology: meeting the global demand for vegetable oils for food, feed, biofuel, and industrial applications. Curr. Opin. Biotechnol. 22, 252-259.

Millar, A. A., Smith, M. A., and Kunst, L. (2000). All fatty acids are not equal: discrimination in plant membrane lipids. Trends Plant Sci. 5, 95-101.

Moreau, R. A., and Stumpf, P. K. (1981). Recent studies of the enzymicsynthesis of ricinoleic acid by developing castor beans. Plant Physiol. 67, 672-676.

Napier, J. A., and Graham, I. A. (2010). Tailoring plant lipid composition: designer oilseeds come of age. Curr. Opin. Plant Biol. 13, 329-336.

Nguyen, H. T., Mishra, G., Whittle, E., Bevan, S. A., Merlo, A. O., Walsh, T. A., and Shanklin, J. (2010). Metabolic engineering of seeds can achieve levels of $\omega-7$ fatty acids comparable with the highest levels found in natural plant sources. Plant Physiol. 154, 1897-1904.

Ohlrogge, J. B., and Jaworski, J. G. (1997). Regulation of fatty acid synthesis. Annu. Rev. Plant Physiol. Plant Mol. Biol. 48, 109-136.

Okuley, J., Lightner, J., Feldmann, K., Yadav, N., Lark, E., and Browse, J. (1994). Arabidopsis FAD2 gene encodes the enzyme that is essential for polyunsaturated lipid-synthesis. Plant Cell 6, 147-158.

Ratcliffe, R. G., and Shachar-Hill, Y. (2006). Measuring multiple fluxes through plant metabolic networks. Plant J. 45, 490-511.

Roughan, P. G., and Slack, C. R. (1982). Cellular-organization of glycerolipid metabolism. Annu. Rev. Plant Physiol. Plant Mol. Biol. 33, 97-132.

Segel, I. H. (1976). Biochemical Calculations. New York: John Wiley \& Sons.

Shockey, J. M., Gidda, S. K., Chapital, D. C., Kuan, J. C., Dhanoa, P. K., Bland, J. M., Rothstein, S. J., Mullen, R. T., and Dyer, J. M. (2006). Tung tree DGAT1 and DGAT2 have nonredundant functions in triacylglycerol biosynthesis and are localized to different subdomains of the endoplasmic reticulum. Plant Cell 18, 2294-2313.

Slack, C. R., Campbell, L. C., Browse, J. A., and Roughan, P. G. (1983). Some evidence for the reversibility of the cholinephosphotransferasecatalysed reaction in developing linseed cotyledons in vivo. Biochim. Biophys. Acta 754, 10-20.

Slack, C. R., Roughan, P. G., and Balasingham, N. (1978). Labeling of glycerolipids in cotyledons of developing oilseeds by [1-C-14] acetate and [2-H-3] glycerol. Biochem. J. 170, 421-433.

Slack, C. R., Roughan, P. G., Browse, J. A., and Gardiner, S. E. (1985). Some properties of cholinephosphotransferase from developing safflower cotyledons. Biochim. Biophys. Acta 833, 438-448.

Snyder, C. L., Yurchenko, O. P., Siloto, R. M. P., Chen, X., Liu, Q., Mietkiewska, E., and Weselake, R. J. (2009). Acyltransferase action in the modification of seed oil biosynthesis. $N$. Biotechnol. 26, 11-16.

Sperling, P., Linscheid, M., Stocker, S., Muhlbach, H. P., and Heinz, E. (1993). In-vivo desaturation of cis-delta-9-monounsaturated to cis-delta-9,12-diunsaturated alkenylether glycerolipids. J. Biol. Chem. 268, 26935-26940.
Stahl, U., Banas, A., and Stymne, S. (1995). Plant microsomal phospholipid acyl hydrolases have selectivities for uncommon fatty-acids. Plant Physiol. 107, 953-962.

Stymne, S., and Stobart, A. (eds.). (1987). Triacylglycerol Biosynthesis. New York: Academic Press.

Stymne, S., and Stobart, A. K. (1984). Evidence for the reversibility of the acyl-coA-lysophosphatidylcholine acyltransferase in microsomal preparations from developing safflower (Carthamus tinctorius L) cotyledons and rat-liver. Biochem. J. $223,305-314$

Thomaeus, S., Carlsson, A. S., and Stymne, S. (2001). Distribution of fatty acids in polar and neutral lipids during seed development in Arabidopsis thaliana genetically engineered to produce acetylenic, epoxy and hydroxy fatty acids. Plant Sci. 161, 997-1003.

Tjellström, H., Yang, Z., Allen, D. K., and Ohlrogge, J. B. (2012). Rapid kinetic labeling of Arabidopsis cell suspension cultures: implications for models of lipid export from plastids. Plant Physiol. 158, 601-611.

van Erp, H., Bates, P. D., Burgal, J., Shockey, J., and Browse, J. (2011) Castor phospholipid:diacylglycerol acyltransferase facilitates efficient metabolism of hydroxy fatty acids in transgenic Arabidopsis. Plant Physiol. $155,683-693$

Vandeloo, F. J., Broun, P., Turner, S., and Somerville, C. (1995). An oleate 12hydroxylase from Ricinus communis $\mathrm{L}$. is a fatty acyl desaturase homolog. Proc. Natl. Acad. Sci. U.S.A. 92, 6743-6747.

Velasco, L., Goffman, F. D., and Becker, H. C. (1998). Variability for the fatty acid composition of the seed oil in a germplasm collection of the genus Brassica. Genet. Resour. Crop Evol. 45, 371-382.

Vichi, S., Pizzale, L., and Conte, L. S. (2007). Stereospecific distribution of fatty acids in triacylglycerols of olive oils. Eur. J. Lipid Sci. Technol. 109, 72-78.

Voelker, T., and Kinney, A. T. (2001). Variations in the biosynthesis of seed-storage lipids. Annu. Rev. Plant Physiol. Plant Mol. Biol. 52, 335-361.

Vogel, G., and Browse, J. (1996). Cholinephosphotransferase and diacylglycerol acyltransferase - substrate specificities at a key branch point in seed lipid metabolism. Plant Physiol. 110, 923-931.

Wallis, J. G., and Browse, J. (2010). Lipid biochemists salute the genome. Plant J. 61, 1092-1106.
Weiss, S. B., and Kennedy, E. P. (1956). The enzymatic synthesis of triglycerides. J. Am. Chem. Soc. 78, 3550-3550.

Weiss, S. B., Kennedy, E. P., and Kiyasu, J. Y. (1960). Enzymatic synthesis of triglycerides. J. Biol. Chem. 235, 40-44.

Weselake, R. J., Taylor, D. C., Rahman, M. H., Shah, S., Laroche, A. Mcvetty, P. B. E., and Harwood, J. L. (2009). Increasing the flow of carbon into seed oil. Biotechnol. Adv. 27, 866-878.

Williams, M., Sanchez, J., Hann, A. C., and Harwood, J. L. (1993). Lipid biosynthesis in olive cultures. J. Exp. Bot. 44, 1717-1723.

Xu, J., Carlsson, A., Francis, T., Zhang, M., Hoffmann, T., Giblin, M., and Taylor, D. (2012). Triacylglycerol synthesis by PDAT1 in the absence of DGAT1 activity is dependent on reacylation of LPC by LPCAT2. BMC Plant Biol. 12, 4. doi:10.1111/j.1365313X.2010.04396.x

Yang, P., Li, X., Shipp, M., Shockey, J., and Cahoon, E. (2010). Mining the bitter melon (Momordica charantia L.) seed transcriptome by 454 analysis of non-normalized and normalized cDNA populations for conjugated fatty acid metabolism-related genes. BMC Plant Biol. 10, 250 doi:10.1186/1471-2229-10-250

Zubr, J., and Matthäus, B. (2002). Effects of growth conditions on fatty acids and tocopherols in Camelina sativa oil. Ind. Crops Prod. 15, 155-162.

Conflict of Interest Statement: The authors declare that the research was conducted in the absence of any commercial or financial relationships that could be construed as a potential conflict of interest.

Received: 09 May 2012; accepted: 14 June 2012; published online: 02 July 2012.

Citation: Bates PD and Browse J (2012)

The significance of different diacylgycerol synthesis pathways on plant oil composition and bioengineering. Front. Plant Sci. 3:147. doi: 10.3389/fpls.2012.00147

This article was submitted to Frontiers in Plant Metabolism and Chemodiversity, a specialty of Frontiers in Plant Science. Copyright $\odot 2012$ Bates and Browse. This is an open-access article distributed under the terms of the Creative Commons Attribution Non Commercial License, which permits non-commercial use, distribution, and reproduction in other forums, provided the original authors and source are credited. 